\title{
A Scalable Pickering Stabilization to Design Cellulose Nanofiber-wrapped Block Copolymer Microspheres for Thermal Energy Storage
}

\author{
Arindam Chakrabarty, Yoshikuni Teramoto*
}

Division of Forest and Biomaterials Science, Graduate School of Agriculture, Kyoto University, Kitashirakawa Oiwake-cho, Sakyo-ku, Kyoto 606-8502, JAPAN

*Corresponding Author Email: teramoto.yoshikuni.3e@kyoto-u.ac.jp

This supporting information has 6 pages containing 7 figures, 1 scheme and 1 table. 


\section{Conductometric titration to calculate DS}

About $5 \mathrm{~g}$ of cNF3 aqueous dispersion $(0.43 \mathrm{wt} \%)$ was taken in a $100-\mathrm{mL}$ glass beaker and diluted with de-ionized water to $40 \mathrm{~g}$. The dispersion was stirred using magnetic bar for few minutes and then conductance was measured using a probe conductivity meter which corresponds to the value at ' 0 ' addition of $\mathrm{AgNO}_{3}$. Then $200-\mu \mathrm{L} \mathrm{AgNO}_{3}(0.01 \mathrm{M})$ was added dropwise and stirred the mixture for 2-3 min before measuring the conductance value. The similar process was repeatedly performed until the plot with following pattern (Fig. S1) was obtained.

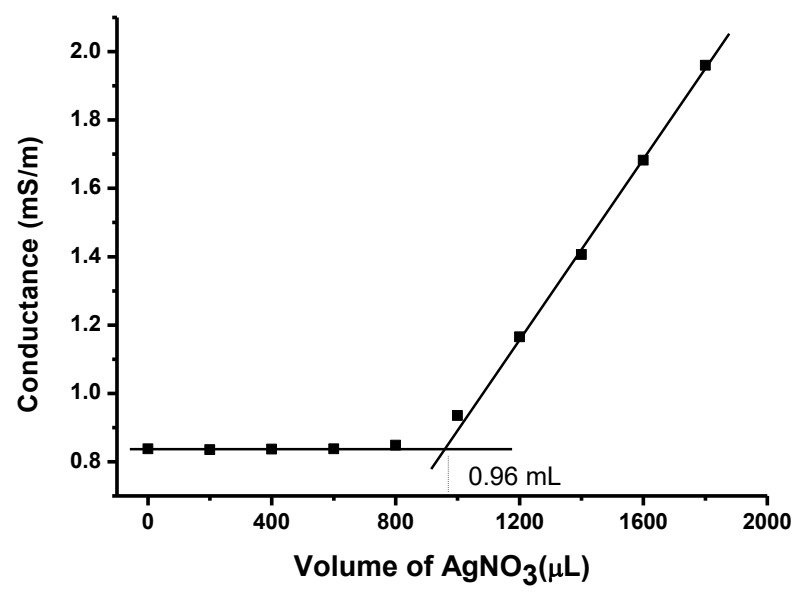

Fig. S1. A representative conductometric titration plot for cNF3 sample.

End point $=0.96 \mathrm{~mL}$

$C_{\mathrm{AgNO} 3}=\frac{0.01 \times 0.96}{1000}=9.6 \times 10^{-6} \mathrm{~mol}$

$C_{\text {cellulose }}=\frac{5 \times 0.0043}{162}=1.3 \times 10^{-4} \mathrm{~mol}$; The molar mass of one anhydroglucose unit is 162.

$\mathrm{DS}=C_{\mathrm{AgN03}} / C_{\text {cellulose }}=0.07$ 


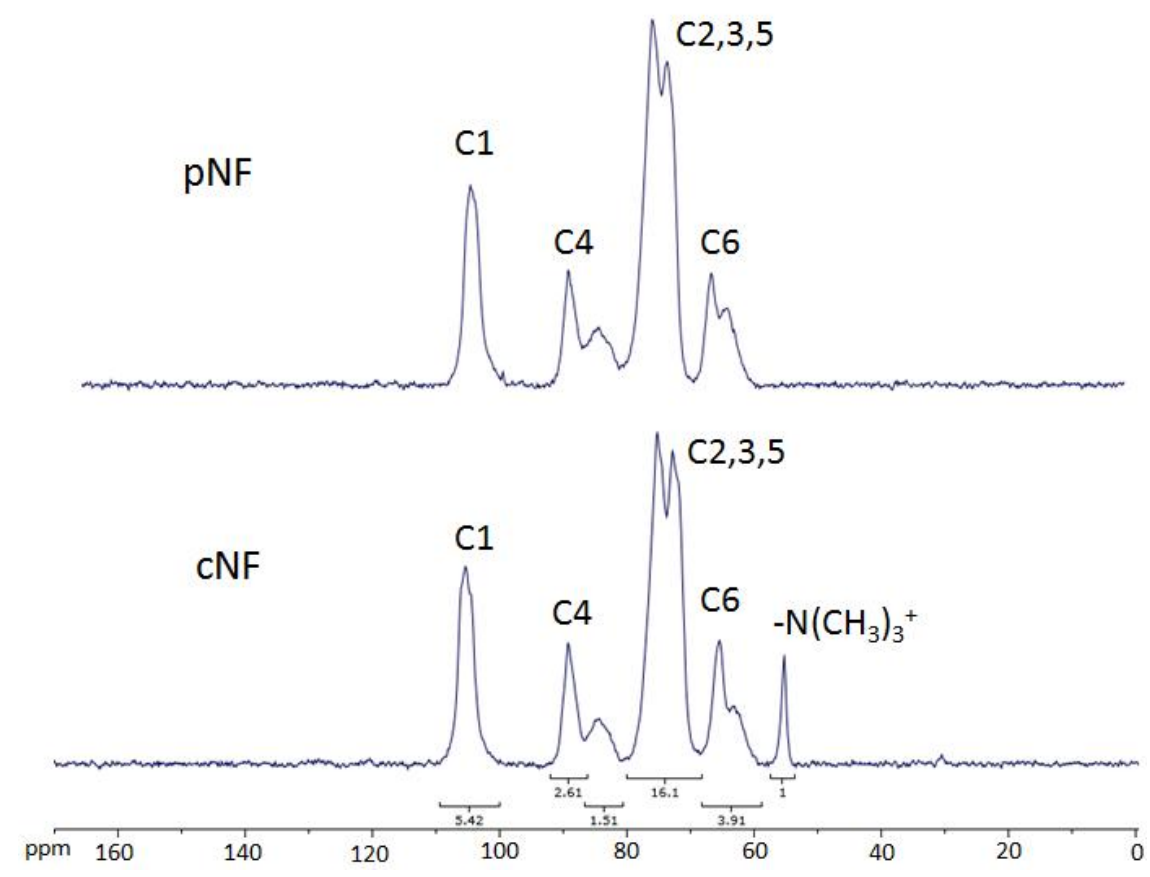

Fig. S2. Solid-state CP/MAS ${ }^{13} \mathrm{C}$ NMR spectra of $\mathrm{pNF}$ and cNF.

$$
D S=\frac{1 / 3}{3.91}=0.08
$$

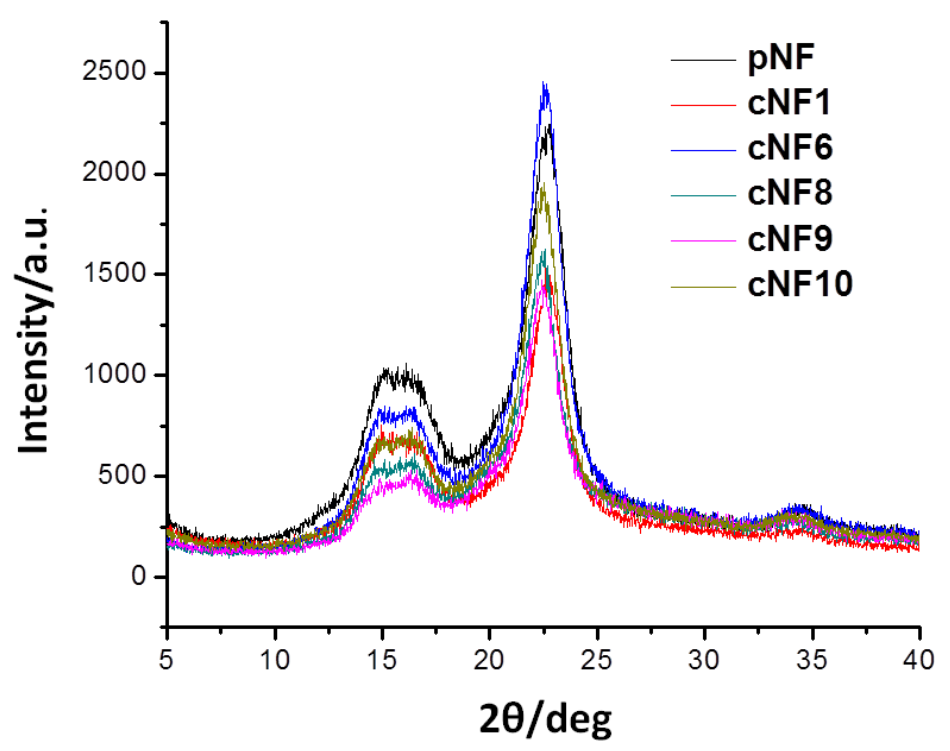

Fig. S3. WAXD profiles of pNF and cNF samples. 


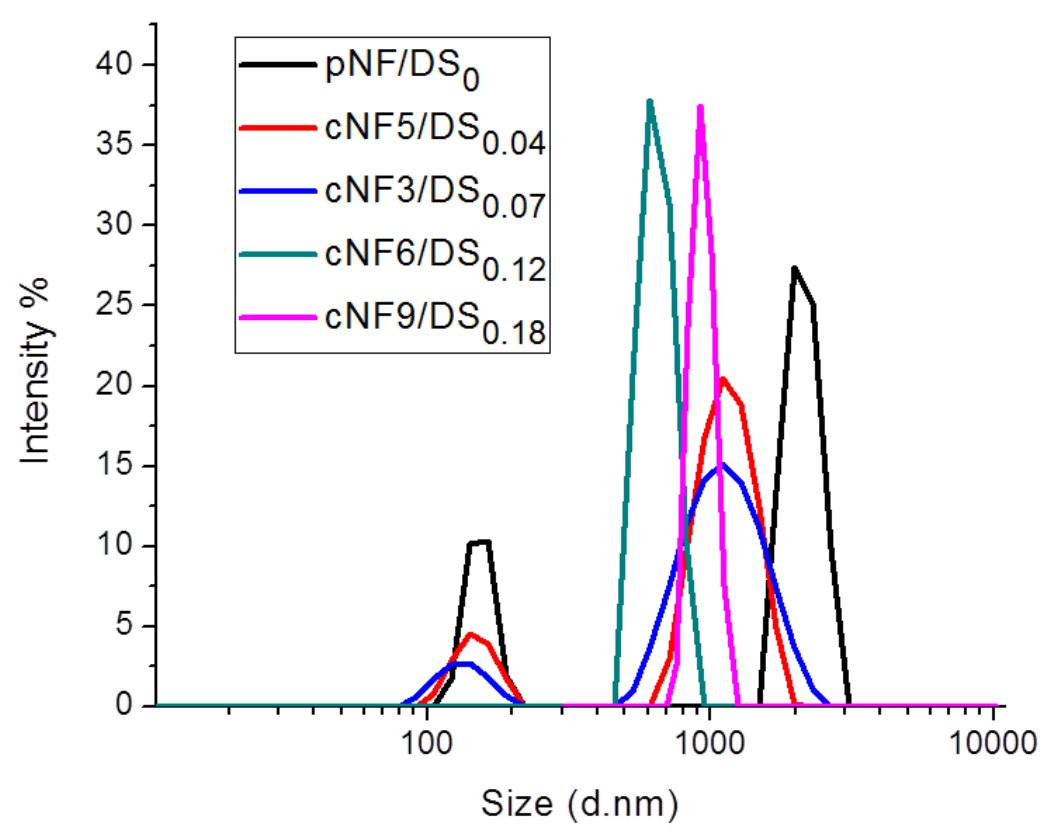

Fig. S4. Particle size distribution profiles of MMA/water Pickering emulsions stabilized by pNF and cNFs.

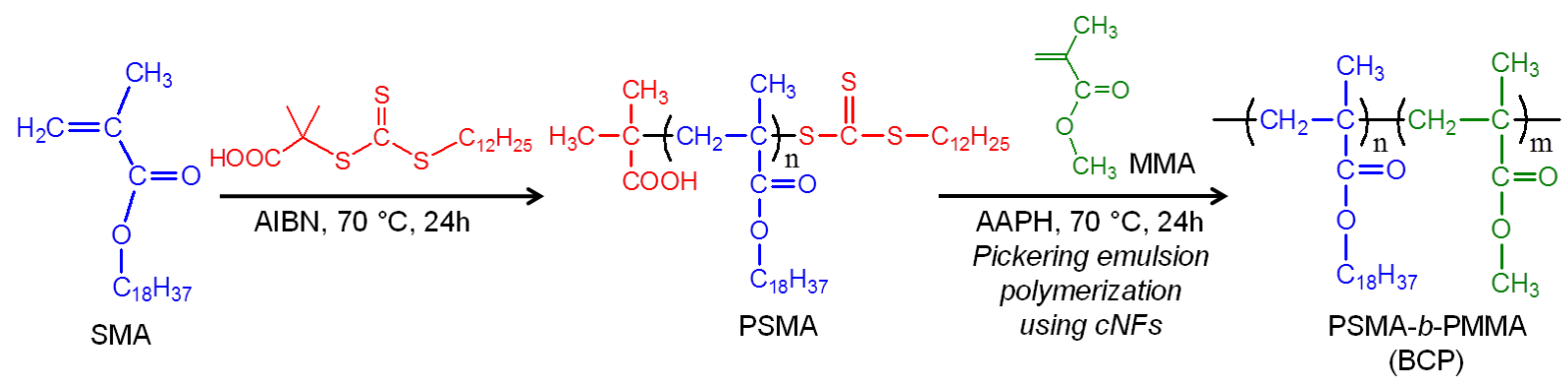

Scheme S1. Synthetic pathway for the preparation of the BCP, PSMA- $b$-PMMA via consecutive RAFT polymerization in solution followed by cNF-stabilized Pickering emulsion. 


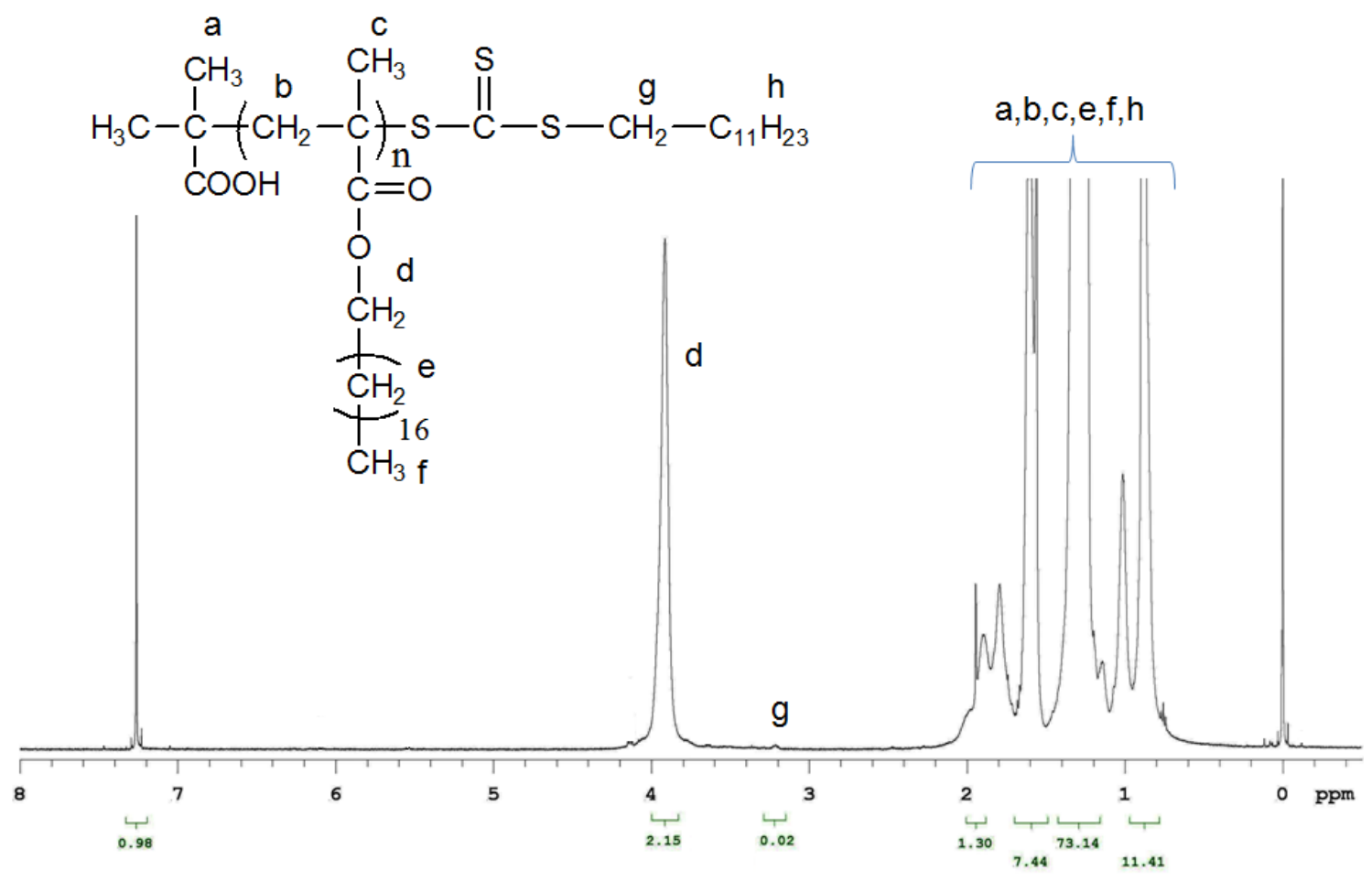

Fig. S5. ${ }^{1}$ H NMR spectrum of PSMA macro-RAFT agent.

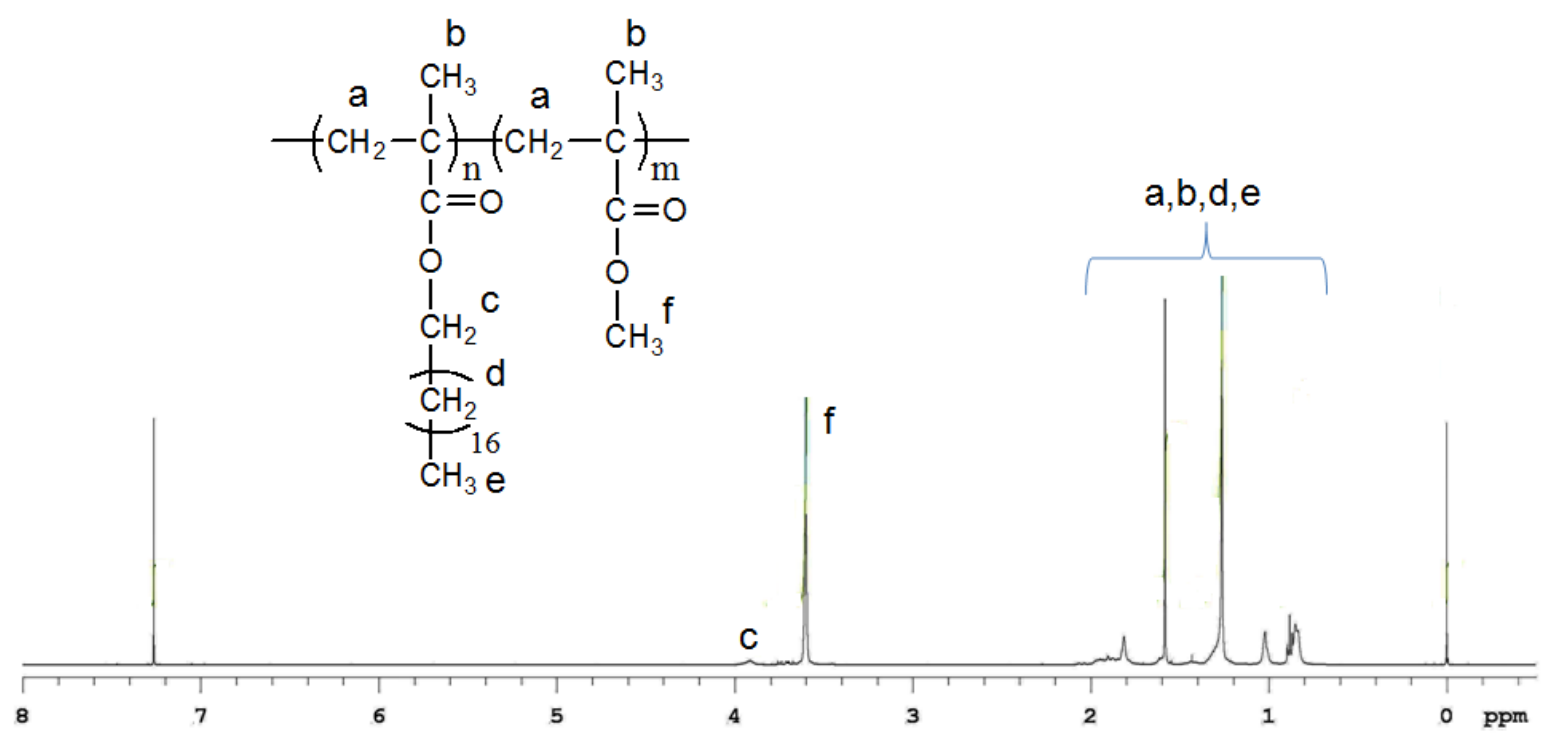

Fig. S6. ${ }^{1} \mathrm{H}$ NMR spectrum of PSMA- $b$-PMMA. 


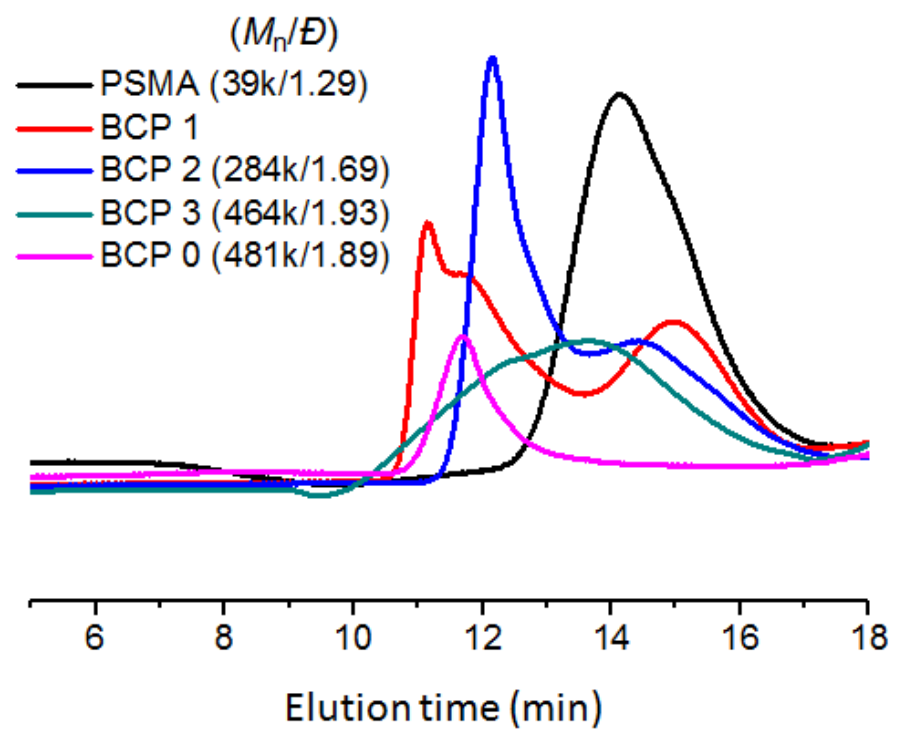

Fig. S7. GPC profiles of PSMA macro-RAFT and the BCPs prepared at different conditions.

Table S1 Results of absorption and release of thermal energy by the pure PSMA and BCPs as measured by DSC at different heating/cooling rate.

Melting

Crystallization

\begin{tabular}{c|ccccc|}
\hline Material & Rate $\left({ }^{\circ} \mathbf{C} / \mathbf{m i n}\right)$ & $\boldsymbol{T}_{\mathbf{m}}\left({ }^{\circ} \mathbf{C}\right)$ & $\Delta \boldsymbol{H}(\mathbf{J} / \mathbf{g})$ & $\boldsymbol{T}_{\mathbf{c}}\left({ }^{\circ} \mathbf{C}\right)$ & $\Delta \boldsymbol{H}(\mathbf{J} / \mathbf{g})$ \\
\hline \multirow{5}{*}{ PSMA } & 5 & 36.7 & 71.1 & 25.8 & -73.0 \\
& 10 & 36.6 & 71.2 & 25.0 & -72.1 \\
& 20 & 36.5 & 75.7 & 24.6 & -74.4 \\
BCP1 & 5 & 34.1 & 0.71 & 24.2 & -0.79 \\
& 10 & 33.7 & 0.67 & 23.5 & -0.55 \\
& 20 & 34.0 & 0.82 & 22.4 & -0.91 \\
BCP2 & 5 & 35.8 & 14.9 & 27.6 & -14.8 \\
& 10 & 35.5 & 14.8 & 26.7 & -14.2 \\
& 20 & 35.2 & 16.2 & 25.4 & -14.9 \\
BCP3 & 5 & 35.4 & 9.6 & 26.7 & -9.8 \\
& 10 & 35.0 & 10.2 & 25.7 & -9.3 \\
& 20 & 35.9 & 10.4 & 24.5 & -10.8 \\
& 5 & 35.1 & 7.4 & 26.5 & -7.6 \\
& 10 & 34.6 & 7.9 & 25.2 & -7.7 \\
& 20 & 34.8 & 10.1 & 23.8 & -9.1 \\
\hline
\end{tabular}

